\title{
How important are the dermatophytes? A clinical and laboratory investigation
}

\author{
DAVID G DAVIES, JOHN DEIGHTON,* WILliAM D PATERSON \\ From the Public Health Laboratory, Carlisle and Cumberland Infirmary, Carlisle
}

SUMMARY Dermatophyte infections were established in $6.9 \%$ of 640 unselected dermatology outpatients attending two district general hospitals in Cumbria over a six-month period. A clinical diagnosis of ringworm was confirmed in the laboratory in $43.1 \%$ of hospital and in $21.8 \%$ of a smaller series of general practice patients. No result of similar surveys have been published before. Even in a major stock-rearing area such as this, domestic pets appear to be a more important source of infection than the farm animal.

The literature concerning dermatophyte infections in Britain is small. In the main it emanates from the Mycology Reference Laboratory, London, reporting fungal isolations from material submitted from all over Britain, though mainly from the Home Counties. ${ }^{1-3} \mathrm{~A}$ changing pattern of infecting agent with some geographical differences is described. Similar investigations in the Bristol and Somerset areas respectively have also been recorded. ${ }^{5}$ However, two main questions remain unanswered by these papers. The proportion of dermatological hospital out-patients suffering from dermatophyte infection and the degree of agreement between the clinical and laboratory diagnosis of fungal infection of the skin both remain unknown. A combined clinical and mycological survey was therefore undertaken for a period of six months on dermatological out-patients attending two district general hospitals in Cumbria. Material from general practice was also solicited so that the prevalence of various infecting agents could be more widely determined.

\section{Patients and methods}

A completed form was sent to the laboratory from every hospital out-patient examined whatever the skin condition present. This provided details of occupation, family history, contact with animals and the clinical diagnosis. A similar return was requested from every patient from general practice in whom a diagnosis of dermatophyte infection had been confirmed.

*Present address: University Department of Surgery, Addenbrooke's Hospital, Cambridge.

Accepted for publication 20 July 1981
In the laboratory, material for fungal examination was screened by direct microscopy and, if positive, cultured on home-made malt extract agar with and without added antibiotics. If fungal material could be demonstrated by either of these methods the result was considered positive, except in the case of Candida albicans where only culture-positive specimens were recorded.

\section{Results}

HOSPITAL OUT-PATIENTS

Records were obtained from 640 patients. Dermatophyte infection was diagnosed clinically in 102 and material submitted for examination. Of these patients, $\mathbf{4 4}$ had positive laboratory results. Hence $6.9 \%$ of all dermatology out-patient were suffering from superficial fungal infection and the clinical diagnosis was correct in $43.1 \%$ of instances.

\section{GENERAL PRACTICE}

Specimens from 124 patients were examined yielding 27 positives. All patients were diagnosed clinically as suffering from dermatophyte infections. Hence $21 \cdot 8 \%$ were correct.

Total survey results

\begin{tabular}{lrr}
\hline Species identification (62 isolates) & & $\%$ \\
\hline Trichophyton mentagraphytes & 21 & $33 \cdot 9$ \\
Candida albicans & 21 & $33 \cdot 9$ \\
Trichophyton rubrum & 9 & $14 \cdot 5$ \\
Microsporum canis & 6 & $9 \cdot 7$ \\
Trichophyton verrucosum & 2 & $3 \cdot 2$ \\
Microsporum gypseum & 1 & 1.6 \\
Microsporum furfur & 1 & $1 \cdot 6$ \\
Epidermophyton floccosum & 1 & 1.6 \\
\hline
\end{tabular}




\section{ANIMAL CONTACT}

A firm history of close animal contact or its complete absence was obtained from 38 patients with fungal infection of the skin. Domestic animals were involved in 21, farm animals in 10 and no animal contact in seven cases. Disordered coats in cats was mentioned in four of the case histories.

\section{Discussion}

The proportion of skin diseases caused by dermatophyte infection is assessed for the first time in Britain as far as is known and is shown to be of significance. The clinical accuracy of a skilled diagnostician in this condition is also impressive. It is possible that not all suspected cases are referred to hospital clinics, perhaps the more obvious ones not progressing beyond the family practitioner. However, in this area, direct contact between the practitioner and the laboratory occurs frequently, many patients being sent directly to the laboratory for fungal examination. Nothing suggests that diagnosis in the dermatophyte infections is obvious or easy.

Again as Cumbria is one of the major British stock-rearing areas it might be supposed that this could influence the results of the investigation. However, the findings indicate that even here, domestic rather than farm animals may constitute the main reservoir of fungal infection. Besides the epidemiological indications the identities of the three main true fungal infecting agents themselves support this unexpected conclusion. $T$ mentagraphytes is occasional in cats, frequent in dogs and occasional in sheep and cattle. ${ }^{6}$ T rubrum appears to be restricted to humans and $M$ canis is usual or frequent in cats and dogs, reported occasionally only in sheep and cattle. It was not possible to examine any animal contacts but loss of hair in domestic cats was mentioned in connection with $M$ canis infections in several patients and the importance of this particular zoonosis has recently been stressed. ${ }^{7}$ Strictly on the laboratory side, the commonest fungal species identified are similar to those reported in earlier surveys but with a somewhat lesser incidence of candidal infection. The occurrence of a double infection with Candida and a true fungus was noted in five patients, all clinically severe. The detection of these by culture may well be important from the therapeutic point of view.

\section{References}

${ }^{1}$ Duncan JT. A survey of fungus diseases in Great Britain; results from the first eighteen months. Br Med J 1945; ii:715-7.

${ }^{2}$ Walker J. The dermatophytoses of Great Britain: report of a three year survey. British Journal of Dermatology and Syphilology 1950;62:239-42.

${ }^{3}$ Murray IG. The changing pattern of dermatophyte infections in the British Isles. Monthly Bulletin of the Ministry of Health Laboratory Service 1966;25:210-4.

4 English MP, Lewis L. Ringworm in the South-West of England, 1960-70, with special reference to onychomycosis. Br J Dermatol 1974;90:67-75.

${ }^{5}$ Greatorex FB. Mycology in Somerset, 1953-75. Med Lab Sci 1978;35:75-80.

${ }^{6}$ Rebell G, Taplin D. Dermatophyte recognition and identification. Florida: University of Miami, 1970.

${ }^{7}$ Dowsett EG, Watson CHS. Communicable disease report. Communicable Disease Surveillance Centre, 1981.

Requests for reprints to: Dr DG Davies, Public Health Laboratory, Cumberland Infirmary, Carlisle, England. 